\title{
QUIRALIDADE EM MOLÉCULAS E CRISTAIS
}

\author{
Ayres Guimarães Dias* \\ Departamento de Química Orgânica, Instituto de Química, Universidade do Estado do Rio de Janeiro, R. S. Francisco Xavier, \\ 524, 20559-900 Rio de Janeiro - RJ, Brasil \\ Renato de Oliveira Soares \\ Fundação Técnico Educacional Souza Marques, Av. Ernani Cardoso, 335, 21310-310 Rio de Janeiro - RJ, Brasil
}

Recebido em 7/1/09; aceito em 13/4/09; publicado na web em 17/9/09

\begin{abstract}
CHIRALITY AT MOLECULES AND CRYSTALS. The present contribution describes some concepts of stereochemistry and chirality in molecules and crystals. This paper also reports on the development of a simple and fast experiment to prepare and recognize conglomerate and true racemate of tartaric acid produced by mechanic mixture of commercial enantiomers and recristalization. Optical activity and melting point of mixtures are also used in the analysis.
\end{abstract}

Keywords: chirality; crystal optical activity; stereochemistry.

\section{INTRODUÇÃO}

A compreensão das relações espaciais presentes em sais e moléculas é fundamental para o entendimento adequado de suas reatividades e propriedades físico-químicas, prioritariamente na química orgânica onde há a possibilidade de formação de várias ligações com diferentes arranjos topológicos. ${ }^{1}$

Existe uma concepção comum aos profissionais da química que produtos opticamente ativos não podem ser gerados de reagentes aquirais sem a intervenção de alguma fonte de quiralidade externa préexistente. A intervenção desta fonte, seja ela qual for, é responsável pela transformação de interações enantioméricas, necessariamente isoenergéticas, em interações diastereoisoméricas, com energias distintas e, por isso, passíveis de diferenciação. ${ }^{2}$ Em outras palavras, a quiralidade não pode ser criada do nada, o que leva a uma intrigante questão sobre a fonte primordial da quiralização. ${ }^{3}$

Os livros de química orgânica em geral são taxativos ao afirmar que enantiômeros possuem as mesmas propriedades físico-químicas como solubilidade, ponto de fusão e reatividade, ficando as diferenças restritas aos respectivos diastereoisômeros. ${ }^{4}$ Este conjunto de premissas não leva em consideração o estado de agregação da espécie, dificultando a contextualizado da própria história da construção dos conceitos estereoquímicos e as propriedades da luz plano polarizada.

A descoberta e o estudo da luz plano polarizada estão associados aos trabalhos desenvolvidos por Biot, ${ }^{5}$ no século XIX, com cristais anisotrópicos como o quartzo. Apesar do conhecimento que alguns líquidos puros e soluções também possuíam atividade óptica o fenômeno ficou associado à natureza cristalina destes compostos, cabendo somente a Pasteur, anos depois, a extensão deste conceito à natureza estrutural das substâncias trabalhando com soluções puras dos respectivos enantiômeros. ${ }^{6,7}$

Todo o conceito moderno de atividade óptica descrito nos principais livros de química orgânica baseia-se na perda parcial de simetria a nível molecular (plano de simetria). ${ }^{8}$ No entanto, as publicações em questão não esclarecem que os elementos de simetria necessários à atividade óptica podem estar ausentes na molécula, porém presentes no seu arranjo cristalino. Podemos destacar, por exemplo, a atividade óptica apresentada pelos cristais de sulfato de etilenodiamônio descrito na Figura 1, contendo plano de simetria a nível molecular. ${ }^{9}$

*e-mail: ayres@uerj.br
Existem duzentos e trinta formas de moléculas e sais se associarem no espaço, sendo sessenta e cinco destas quirais. Moléculas quirais necessariamente cristalizam em uma destas formas, porém algumas moléculas aquirais também são capazes de se associar em um destes arranjos. ${ }^{10}$ Esta particularidade explica o porquê que a molécula descrita na Figura 1, na forma de cristal apresenta atividade óptica.
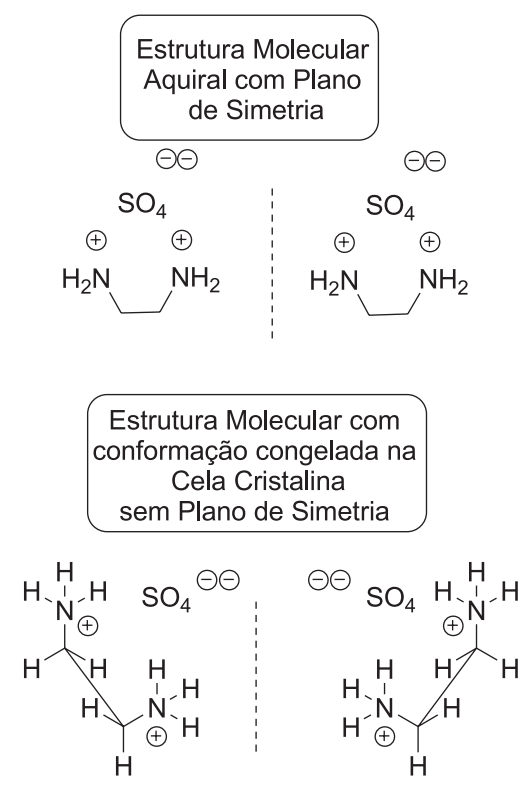

Figura 1. Exemplo de molécula aquiral com estrutura cristalina assimétrica

\section{DISCUSSÃO}

Para contextualizar a questão da quiralidade associada à forma de agregação molecular este trabalho sugere, após o término das discussões envolvendo o tópico estereoquímica, uma breve apresentação da questão do desvio da luz plano polarizada de cristais oriundos de moléculas ou sais detentores de plano de simetria. O exemplo descrito na Figura 1 associado ao clorato de potássio e o quartzo $\left(\mathrm{SiO}_{4}\right)$ serve para explicar a ocorrência natural de luz plano polarizada em um ambiente supostamente aquiral, facilitando tremendamente o entendimento dos primeiros experimentos envolvendo este tema. ${ }^{10}$ 
A resolução de Pasteur é um belíssimo exemplo para aprofundarmos a questão da assimetria molecular e de seus agregados cristalinos e suas experiências são estudadas até os dias atuais. ${ }^{11,12} \mathrm{O}$ ácido racêmico precipitado nos tonéis de vinícolas do sul da França não apresentava atividade óptica, mas possuía fórmula molecular e semelhança química com o ácido tartárico. Observando cuidadosamente as amostras, Pasteur reconheceu a quiralidade nos cristais e após a coleta manual de cada uma das populações submeteu a solução de cada um destes à luz plano polarizada observando a total coincidência de valores em sentidos opostos. ${ }^{13} \mathrm{O}$ grande marco diferenciador neste experimento foi a manutenção da atividade óptica após a dissolução dos compostos, pois até o momento os pesquisadores sempre tinham observado a perda da atividade óptica quando estes eram dissolvidos ou fundidos como, por exemplo, o quartzo e o clorato de sódio. Esta descoberta levou à questão da atividade óptica ao nível molecular e acabaria por dar contribuições fundamentais para o postulado de Le Bel e Van't Hoff sobre a natureza tetraédrica do átomo de carbono. ${ }^{14}$

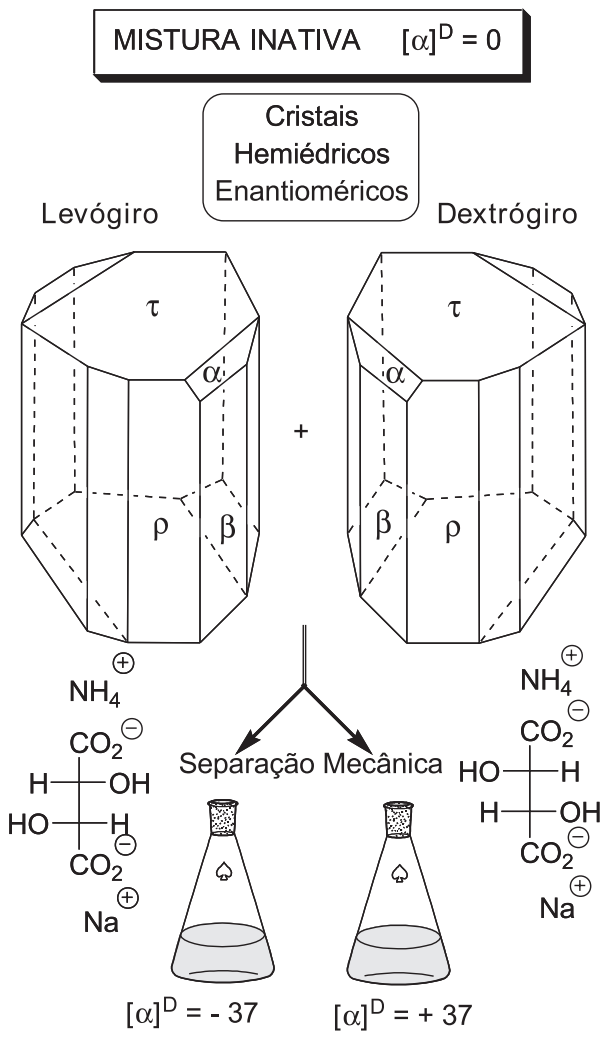

Moléculas Enantioméricas

Figura 2. Estrutura cristalina e atividade óptica do conglomerado isolado por Pasteur

Como para os químicos orgânicos o átomo de carbono é o motivo último de estudos, ao longo dos anos a questão da atividade óptica associada à estrutura cristalina foi sendo deixada de lado, uma vez que as observações das moléculas orgânicas passaram a ser feitas em solução. Tendo a atividade óptica deixado de ser um problema na caracterização de estereoisômeros, as dificuldades envolvendo a compreensão da relação entre a natureza estrutura cristalina e suas propriedades físicas migraram para o ponto de fusão.

$\mathrm{O}$ ponto de fusão, $\mathrm{PF}$, é uma ferramenta analítica fundamental na caracterização inequívoca de moléculas orgânicas, funcionando também como um excelente indicativo do seu grau de pureza. Mesmo que o desenvolvimento dos equipamentos analíticos tenha diminuído a sua importância, a determinação do PF ainda é capaz de fornecer informações importantes rapidamente a baixo custo, justificando sua presença em todos os livros textos de química orgânica experimental. ${ }^{15}$

A técnica, no entanto, apresenta particularidades normalmente pouco discutidas nos livros texto como, por exemplo, as variações de PF em função da maneira como o cristal foi formado. ${ }^{16}$ Esse fenômeno é de grande relevância para a indústria farmacêutica, devido à variabilidade observada na absorção de fármacos associada à forma cristalina da substância (polimorfismo). ${ }^{17}$ Alguns compostos são capazes de cristalizar em diferentes formas cristalinas levando, em alguns casos, a variações de $\mathrm{PF}$ superiores a $40{ }^{\circ} \mathrm{C}$, como o ácido 2-hidroxinicotínico e o 3 -aminofenantreno apresentados na Figura $3 .{ }^{18}$

O PF é a temperatura na qual uma substância muda do estado sólido para a forma líquida, sendo totalmente dependente de fatores como a energia envolvida na formação de sua rede cristalina. Diferentes tipos de redes estão relacionados com valores distintos de energia, justificando as diferentes temperaturas observadas no PF para um mesmo composto., ${ }^{1,18,19}$<smiles>O=C(O)c1cccnc1O</smiles><smiles>Brc1cccc2ccccc12</smiles>

$$
\begin{array}{llll}
\text { PF } \alpha & 259-261^{\circ} \mathrm{C} \text { (dec.) } & \text { PF } \alpha & 6,2{ }^{\circ} \mathrm{C} \\
\text { PF } \beta & 301-302^{\circ} \mathrm{C} \text { (dec.) } & \text { PF } \beta & 2-3{ }^{\circ} \mathrm{C}
\end{array}
$$<smiles>Nc1ccc2ccc3ccccc3c2c1</smiles>

$$
\text { PF } \alpha 143^{\circ} \mathrm{C}
$$$$
\text { PF } \beta \quad 87,5^{\circ} \mathrm{C}
$$<smiles>CC(C)C(=O)O</smiles>

PF $\alpha 7,7^{\circ} \mathrm{C}$

PF $\beta 16^{\circ} \mathrm{C}$

PF $\gamma 5,5^{\circ} \mathrm{C}$<smiles>C[C@@]12CCCC(=O)N1C[C@@H]1CCCN3CCC[C@H]2[C@H]13</smiles>

PF $\propto 26-77^{\circ} \mathrm{C}$

PF $\beta 87^{\circ} \mathrm{C}$

PF $\delta 84^{\circ} \mathrm{C}$<smiles>O=C(O)C(=O)O</smiles>

PF $\alpha 189,5^{\circ} \mathrm{C} ;$ PF $\beta 182{ }^{\circ} \mathrm{C}$ (anidro) PF $\beta 101,5^{\circ} \mathrm{C}$ (hidratado)

\section{$\alpha, b, \delta:$ Formas cristalinas distintas}

Figura 3. Exemplos de moléculas com diferentes formas cristalinas e PF

Para tornar a questão sobre o PF ainda mais complexa os estereoisômeros apresentam um comportamento diferenciado, apesar de possuírem a mesma fórmula molecular. Enquanto os diastereoisômeros apresentam diferentes PF os enantiômeros, como esperado, apresentam os mesmos valores. No entanto, para a mistura de quantidades iguais dos enantiômeros (racemato), podemos observar discrepâncias surpreendentes. $\mathrm{O}$ racemato funde de maneira semelhante a uma substância pura apresentando, em um número significativo de casos, PF e solubilidades diferentes dos enantiômeros puros. ${ }^{1}$

Os racematos podem cristalizar de três formas distintas, sendo a compreensão destas de importância fundamental para os estudos envolvendo sua resolução, Tabela 1. Os racematos podem cristalizar 
Tabela 1. Formas cristalinas e propriedades de misturas enantioméricas

\begin{tabular}{|c|c|c|c|c|}
\hline Cristais de enantiômeros & Composição & PF & {$[\alpha]^{\mathrm{D}}$ cristal } & Solubilidade \\
\hline Conglomerado & Mistura 1:1de cristais (+) e (-) & Menor que (+) e (-) puros & --- & $\begin{array}{l}\text { Semelhante a } \\
(+) \text { e }(-) \text { puros }\end{array}$ \\
\hline Composto racêmico & Cela Cristalina $1: 1 ;(+/-)$ & Sem correlação & Zero & $\begin{array}{l}\text { Semelhante a } \\
(+) \text { e (-) puros }\end{array}$ \\
\hline Pseudorracemato & $\begin{array}{l}\text { Mistura de } \\
(+/-) ;(+) ;(-)\end{array}$ & Igual ou próximo (+) e (-) puros & Sem correlação & $\begin{array}{l}\text { Semelhante a } \\
(+) \text { e (-) puros }\end{array}$ \\
\hline
\end{tabular}

* (+/-): Cela cristalina composta de enantiômeros. (+/+) ou (-/-): celas cristalinas compostas por moléculas de mesma quiralidade.

na forma de conglomerados, compostos racêmicos (também conhecidos como racematos verdadeiros) e pseudorracematos (também denominados como sólidos racêmicos). ${ }^{1}$

O conglomerado constitui-se de uma mistura mecânica em quantidades equimoleculares de cristais enantioméricos. O ponto de fusão observado é sempre mais baixo que os dos respectivos enantiômeros puros. Nos compostos racêmicos, os enantiômeros possuem maior afinidade pelo seu antípoda que por outra molécula de mesma estereoquímica, e a substância cristaliza conjugada na proporção 1:1 em uma chamada célula elementar. ${ }^{12,19}$ Esta forma possui solubilidade e pontos de fusão diferenciados, podendo estes ser de valor maior ou menor que o dos antípodas puros. Caso o composto racêmico contenha um ligeiro excesso de um de seus componentes, observamos uma faixa e o abaixamento de seu ponto de fusão, tendo o excesso agido como uma impureza do cristal.

Nos pseudorracematos não existe diferença de afinidade entre a molécula e seu antípoda. Seu cristal possui uma distribuição aleatória coexistindo associações de enantiômeros (R/S) e homoquirais (R/R ou $\mathrm{S} / \mathrm{S})$. Um pequeno excesso de um dos componentes altera de maneira quase imperceptível o PF, Tabela $1 .^{1,18,19}$

Visando a consolidação dos conceitos envolvendo a natureza da agregação molecular e as propriedades físico-químicas associadas, como solubilidade, atividade óptica e ponto de fusão de espécies enantioméricas, esse trabalho propõe um experimento simples envolvendo a medição de PF dos enantiômeros comerciais do ácido tartárico e a preparação de seus respectivos conglomerados e racematos. A escolha desta molécula fundamenta-se na sua importância histórica, disponibilidade comercial e baixo custo. ${ }^{20}$

A solubilidade e os dados físico-químicos dos ácidos tartáricos são facilmente obtidos na literatura e forneceram a base de dados necessária ao experimento proposto. ${ }^{21} \mathrm{O}$ aluno deve fazer as medições de PF e atividade óptica nos ácidos puros e, posteriormente, preparar um racemato misturando quantidades equimoleculares dos ácidos (+) e (-). Após a obtenção do PF dos ácidos, uma recristalização da mistura em microescala em etanol e secagem dos cristais, os valores obtidos são comparados com os da literatura. O ponto de fusão do produto recristalizado difere da mistura mecânica por cerca de $30^{\circ}$, fundindo como uma substância pura entre 205 e $206^{\circ} \mathrm{C}$, comprovando a mudança do estado de agregação do cristal, Tabela $2 .{ }^{21} \mathrm{~A}$ ausência de atividade óptica do racemato verdadeiro produzido, entrada 3 da Tabela 2, comprova a natureza do cristal obtido, enquanto o ácido puro apresentou os valores descritos na literatura, entradas 1 e 2, Tabela $2 .{ }^{21}$ A comparação de seus PF aliado aos dados de atividade óptica nos permite caracterizar todas as formas de cristalização obtidas.

\section{CONSIDERAÇÕES FINAIS}

O distanciamento do ensino de química orgânica de questões envolvendo a natureza das estruturas cristalinas em moléculas e sais tem gerado uma série de dificuldades no aprendizado de este-
Tabela 2. Propriedades dos racematos de ácido tartárico preparados

\begin{tabular}{|c|c|c|c|}
\hline Entrada & Produto & $\mathrm{PF}$ & {$[\alpha]^{\mathrm{D}}$ solução } \\
\hline 1 & $\begin{array}{l}\text { Ac. (+)tartárico } \\
\text { (AT) }\end{array}$ & $\begin{array}{c}171-177^{\circ} \mathrm{C} \\
\text { Lit. }\left(171-177^{\circ} \mathrm{C}\right)^{18,21}\end{array}$ & $\begin{array}{c}\quad+12,7 \\
\text { Lit. }+12,7^{18,21} \\
\left(\mathrm{c}=17,4 ; \mathrm{H}_{2} \mathrm{O}\right)\end{array}$ \\
\hline 2 & $\begin{array}{l}\text { Ac. (-)tartárico } \\
\text { (AT) }\end{array}$ & $\begin{array}{c}171-177^{\circ} \mathrm{C} \\
\text { Lit. }\left(171-177^{\circ} \mathrm{C}\right)\end{array}$ & $\begin{array}{c}-12,7 \\
\text { Lit. }+12,7^{18,21} \\
\left(\mathrm{c}=17,4 ; \mathrm{H}_{2} \mathrm{O}\right)\end{array}$ \\
\hline 3 & $\begin{array}{l}\text { AT composto } \\
\text { racêmico }\end{array}$ & $\begin{array}{l}205-206^{\circ} \mathrm{C}^{*} \\
\text { Lit. }\left(206^{\circ} \mathrm{C}\right)^{10}\end{array}$ & Zero \\
\hline 4 & $\begin{array}{l}\text { AT pseudor- } \\
\text { racemato }\end{array}$ & $175-190^{\circ} \mathrm{C}$ & --- \\
\hline
\end{tabular}

* Recristalizado de uma solução $17 \%$ em etanol a $-25^{\circ} \mathrm{C}$.

reoquímica e no entendimento de algumas propriedades associadas ao estado de agregação da matéria, como a atividade óptica de sólidos e ponto de fusão. Neste trabalho apresentamos uma breve discussão sobre o assunto e um experimento simples, de fácil execução e baixo custo, capaz de promover e aparelhar discussões envolvendo a simetria entre cristais e moléculas. Acreditamos que, longe de tornar o tema ainda mais complexo, a discussão destes pormenores permite o pleno entendimento sobre a natureza da luz plano polarizada, sua interação com a matéria no estado sólido ou em solução. A discussão sobre o estado da matéria acaba por permitir a introdução da questão envolvendo a variabilidade de ponto de fusão apresentada por algumas substâncias puras e o tema polimorfismo.

\section{PARTE EXPERIMENTAL}

Os pontos de fusão foram determinados em aparelho Thomas Hoover, capillary Melting Point Apparatus, e devidamente corrigidos. A atividade óptica foi medida em um polarímetro circular modelo Polax e as recristalizações efetuadas com solventes P.A. comerciais disponíveis no país.

\section{Recristalização}

Em um tubo de ensaio de $10 \mathrm{~mL}$ misturar 0,250 g de (-)ácido tartárico e 0,250 g de (+)ácido tartárico. Dissolver a mistura sólida em $5 \mathrm{~mL}$ de etanol previamente aquecido e deixar a solução em repouso à temperatura de $-20{ }^{\circ} \mathrm{C}$ (freezer) por um período de 7 a 10 dias. O precipitado obtido deve ser decantado cuidadosamente e o sólido seco sob vácuo, dando origem a um sólido branco com massa oscilando entre 0,200 a $0,380 \mathrm{~g}$, com ponto de fusão $205-206^{\circ} \mathrm{C}$ (p.f. lit. $\left..^{18,21}=205-206{ }^{\circ} \mathrm{C}\right)$. 


\section{Atividade óptica}

Dissolver 500,0 mg (2,3 mmol) de ácido (+) ou (-)tartárico em 5 $\mathrm{mL}$ de água destilada e diluir analiticamente em balão volumétrico de $10 \mathrm{~mL}$. O mesmo procedimento deve ser executado para a uma mistura contendo $250,0 \mathrm{mg}$ do conglomerado ou ácido tartárico de PF $205-206^{\circ}$ C. As soluções devem ser observadas em polarímetro e os valores obtidos devem ser comparados entre si e correlacionados com os PF obtidos.

\section{AGRADECIMENTOS}

Aos Profs. F. Louchard e M. de M. Silva pela leitura e sugestões e à FAPERJ pelo financiamento.

\section{REFERÊNCIAS E NOTAS}

1. Smith, M. B.; March, J.; Advanced Organic Chemistry, $5^{\text {th }}$ ed., John Wiley \& Sons: New York, 2000, cap. 13; Eliel, E. L., Wilen, S. H.; Stereochemistry of Organic Compounds, John Wiley \& Sons: New York, 1994, cap. 9; Lima, V. L. E.; Quim. Nova 1997, 20, 657.

2. Coelho, F.; Cadernos Temáticos de Química Nova na Escola 2001, n. 3, 23; Pessine, F. B. T.; de Andrade, J. B.; Quim. Nova 1999, 22, 454; Lipkowitz, K. B.; Nylor, T.; Anliker, K. S.; J. Chem. Educ. 2000, 77, 305.

3. Brewster, J. H.; J. Chem. Educ. 1986, 63, 667.

4. Clayden, J.; Greeves, N.; Warren, S.; Wothers, P.; Organic Chemistry, Oxford: New York, 2007; Vollhardt, K. P. C.; Schore, N. E.; Organic Chemistry - Structure and Function, $4^{\text {th }}$ ed., W. H. Freeman and Company: New York, 2003; Solomons, G.; Fryhle, C., Organic Chemistry, $7^{\text {th }}$ ed., John Wiley and Sons: New York, 2000; Caswell, L.; GarciaGaribai, M. A.; Scheffer, J. R.; Trotter, J.; J. Chem. Educ. 1970, 70, 785; Constantino, M. G.; Química Orgânica/Curso Básico Universitário, LTC: São Paulo, 2008, vol. 2.

5. Biot, J. B.; Mem. Acad. Sci. Inst. 1819, 2, 41; Ault, A.; J. Chem. Educ. 2004, 81, 1605.

6. Pasteur, L.; Ann. Chim. Phys. 1848, 24, 442; Pasteur, L.; Compt. Rend. $\mathbf{1 8 4 9}, 28,477$

7. Uma importante discussão histórica envolvendo os primeiros experimentos com cristais opticamente ativos foi recentemente aberta na literatura: Jacoby, M.; Chem. Eng. News 2008, 18, 38; Kahr, B. Gurney, R. W.; Chem. Rev. 2001, 101, 893.

8. Lloyd-Williams, P.; Giralt, E.; J. Chem. Educ. 2003, 80, 1178 e ref. 4.

9. Koby, L.; Ningappa, J. B.; Dakessian, M.; Cuccia, L. A.; J. Chem. Educ. 2005, 82, 1043.
10. Suh, II-H.; Park, K. H.; Jensen, W. P.; Lewis, D. E.; J. Chem. Educ. 1997, 74,800; Chvátal, M.; Mineralogia para Principiantes: Cristalografia, Sociedade Brasileira de Geologia: Rio de Janeiro, 2007; Qian, R-Y.; Botsaris, G. D.; Chem. Eng. Sci. 1998, 53, 1745.

11. Roberts, R. M.; Descobertas Acidentais em Ciências, Papirus: Campinas, 1995, cap.12.; Andrade-Gamboa, J.; J. Chem. Educ. 2007, 84, 1783.

12. Wheeler, K. A.; Grove, R. C.; Davis, R. E.; Kassel, W. S.; Angew. Chem. I.E.E. 2008, 47, 78; Pérez-Garcia, L.; Amabilino, D. B.; Chem. Soc. Rev. 2002, 31, 342.

13. Pasteur teve a "sorte" de conseguir cristais de formato enantiomérico capazes de serem reconhecidos a olho nu. O mais comum nesse tipo de cristalização é a formação de cristais isofórmicos, porém com atividade óptica distinta, passíveis de diferenciação apenas pela interação com a luz plano polarizada. Um exemplo interessante desta diferenciação é descrito detalhadamente na ref. 9.

14. Snelders, H. A. S.; J. Chem. Educ. 1974, 51, 1; Le Bel, J. A.; Bull. Soc. Chim. Paris 1874, 22, 333; Van't Hoff, J. H.; Arch. Neerl. Sci. Exacts Nat. 1874, 9, 445 .

15. Vogel, A. I.; Vogel's Textbook on Experimental Organic Chemistry, Longmann: Essex, 1989; Dias, A. G.; Costa, M. A.; Guimarães, P. I. C.; Guia Prático de Química Orgânica, Interciência: Rio de Janeiro, 2004, vol. I.

16. Moseley, C. G.; J. Chem. Educ. 1989, 66, 1063; Tiers, G. V. D.; J. Chem. Educ. 1990, 67, 258.

17. Barreiro, E. J.; Fraga, C. A. M.; Química Medicinal: As Bases Moleculares da Ação dos Fármacos, $2^{\mathrm{a}}$ ed., Artmed: Porto Alegre, 2001, cap. 5; Barreiro, E. J.; Ferreira, V. F.; Costa, P. R. R.; Quim. Nova 1997, 20, 647; Santos, A. R.; Pinheiro, A. C.; Sodero, A. C. R.; Cunha, A. S.; Padilha, M. C.; Sousa, P. M.; Fontes, S. P.; Veloso, M. P.; Fraga, C. A. M.; Quim. Nova 2007, 30, 125; Anderson, G. L.; Page, S. T.; J. Chem. Educ. 2004, 81, 971; Murakami, H.; Top. Curr. Chem. 2007, 269, 273.

18. Weast, R. C., ed.; Handbook of Chemistry and Physics, CRC: NewYork, $67^{\text {th }}$ ed., 1986.

19. Von Zelewsky, A.; Stereochemistry of Coordination Compounds, John Wiley \& Sons: New York, 1996; Jacques, J.; Collet, A.; Wilen, S. H.; Enantiomers, Racemates and Resolutions, Krieger Publishing Company: Malabar, 1994.

20. Recentemente um exemplo único de cela com uma razão entre os antípodas diferente de 1:1 foi descrita em Tabora, J. E.; Corry, J.; Osifchin, R.; Lepore, J. V.; Davidson, O. A.; Thien, M. P.; Fluid Phase Eq. 2007, 258, 140.

21. Ácido-(+)-tartárico: 100 g; U\$ 21.00 e Ácido-(-)-tartárico: 25g; U\$ 31.30; Aldrich Catalogue, Aldrich Company; 2007-2008; $[\alpha]^{\mathrm{D}}=12,7$ $\left(\mathrm{H}_{2} \mathrm{O} ; \mathrm{c} 17,4\right)$. 